\title{
Geographical clustering of acute adult leukaemia in the East Anglian region of the United Kingdom: a registry-based analysis
}

\author{
P Badrinath, N E Day, D Stockton
}

An ecological analysis by Alexander et $a l^{1}$ observed a small but significant excess of leukaemia excluding chronic lymphoid leukaemia in estuarine wards (relative risk $=1.12$, $95 \%$ CI $1.01,1.25)$. This analysis was confined to cases diagnosed during 1984-1986. Using regional leukaemia incidence data for 19811994, we performed a clustering study based on postcode districts ${ }^{2}$ of address at diagnosis to test this hypothesis further. We did not have population denominator data by postcode district, age, sex, and calendar year for the study period. We therefore adopted a case-control approach within the East Anglian (EA) cancer registry, equivalent to proportional incidence analyses. $^{3}$

\section{Methods}

The EA cancer registry has collected population-based cancer incidence data in the region since 1971, with postcodes of residence given since 1981. From the registry we abstracted information on all cases of acute leukaemia (ICD 9: 2040, 2050, 2060, 2070,
2080), aged over 14 years diagnosed between 1981-1994. For each case we selected 10 nonhaematological malignancies (ICD 9: 140$199)$ as controls matched for sex, age ( $+/-$ two years) and year of diagnosis ( $+/-$ five years). The null hypothesis was that leukaemia occurs with equal incidence across the postcode districts in the region. Conditional upon the total occurrence of cases and controls within a postcode district, the number of cases in a postcode district follows a binomial distribution with proportion parameter equal to the ratio of the number of cases to the total number of cases and controls in the study. We calculated the expected leukaemia cases in each postcode district and plotted the observed/expected ratios on a map and derived the test statistic (observed-expected/standard error) and two sided probabilities.

\section{Results}

Eight hundred and twenty six cases of acute leukaemia and 7711 matched controls were included in the analysis. Figure 1 shows the

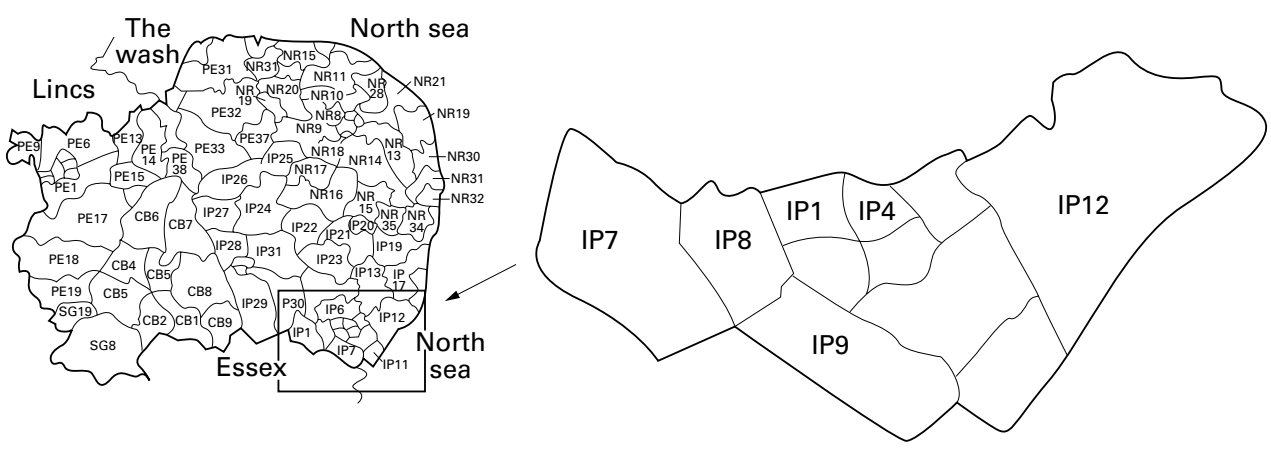

East Anglian Cancer Intelligence Unit, Department of Community Medicine, Institute of Public Health, University of Cambridge, Robinson Way, Cambridge CB2 2SR

Correspondence to: Professor Day.

Accepted for publication 31 October 1998

$\begin{array}{lcl} & \text { Observed } & \text { O/E } \\ \text { CB6 } & 17 & 1.77^{*} \\ \text { IP1 } & 16 & 1.62^{*} \\ \text { IP4 } & 17 & 1.85^{* *} \\ \text { IP7 } & 9 & 1.98^{*} \\ \text { IP8 } & 7 & 2.89^{*} \\ \text { IP9 } & 9 & 2.11^{*} \\ \text { IP12 } & 16 & 2.40^{* *} \\ \text { IP28 } & 12 & 1.80^{*} \\ \text { * } \leq 0.05,{ }^{*} p \leq 0.01, \\ { }^{* * *} \leq \leq 0.001\end{array}$

Figure 1 Acute adult leukaemia clustering in East Anglia 1981-94. 
postcode districts with a nominally statistically significant excess of leukaemia cases in the region. Of the eight postcode districts thus identified with a nominal excess of leukaemia, there is a striking degree of geographical clustering of cases in the IP postcode districts (IP1,4,7,8,9,12). These postcode districts are adjacent to the estuaries of the Stour (IP9), the Orwell (IP1,4,8,9), Deben (IP12), and Ore (IP12). The excess in this cluster of postcode districts was seen equally in men (2.12), women (1.78), in those aged below (1.94) or above (2.00) 65 years and in both periods examined (<1988:1.61, $\geqslant 1988: 2.27)$. The later time period excluded the period considered by Alexander et al. ${ }^{1}$

\section{Discussion}

The major estuaries around the Norfolk and Suffolk coast are those of the Stour, Orwell, Deben, and Ore. The definition of an estuary used by Alexander et $a l^{1}$ is lacking in precision ("all river mouths with a substantial amount of mud marked on the OS map"), but it is doubtful whether the river mouths of the Yare, at Yarmouth or of the Waveney at Lowestoft satisfy the criterion. Our results therefore confirm the findings of an earlier ecological analysis that observed an estuarine excess. The relative excess, however, is markedly larger than was seen earlier, with $\mathrm{O} / \mathrm{E}$ ratios of over 2, compared with the relative risk of 1.12 reported by Alexander et al.
The authors of the previous analysis attribute the estuarine excess to heavy metals and radio actinides found in the estuarine slit. There is no evidence that the population would suffer any appreciable exposure from such a source. The estuaries involved, however, are noteworthy for the extent of maritime activities, both recreational and occupational with boat building and repair being important forms of employment (Dr P Baxter, personal communication). Both recreational and occupational activity would involve potential exposure to resins, solvents, paints and petroleum products, which have been shown both in East Anglia ${ }^{4}$ and elsewhere ${ }^{5}$ to be risk factors for acute leukaemia. In conclusion our study has confirmed the previous finding, but we propose an alternative mechanism for this observed excess.

We would like to thank the staff of the East Anglian cancer registry for providing the data.

1 Alexander FE, Cartwright RA, McKinney PA, et al. Leukaemia incidence, social class and estuaries: an ecological mia incidence, social class and estuaries: an
analysis. F Public Health Med 1990;12:109-17.

2 Swerdlow AJ. Cancer incidence data for adults. In: Elliot P, Cuzick J, English D, et al. Geographical and environmental epidemiology: Methods for small area studies. Oxford: Oxford University Press, 1992

3 IARC scientific publications No 47. Cancer incidence in Singapore 1968-1977. Appendix F. Lyon: International Agency for Research on Cancer, 1983.

4 Badrinath P. East Anglian case control study of acute leukaemia in adults. [PhD Thesis.] Cambridge: University of Cambridge, 1997.

5 Linet MS, Cartwright RA. The leukaemias. In: Schottenfeld D, Fraumeni JF Jr, eds. Cancer epidemiology and prevention. New York: Oxford University Press, 1996:841-92. 